\title{
Mammary Chain Irradiation in Left-Sided Breast Cancer: Can We Reduce the Risk of Secondary Cancer and Ischaemic Heart Disease with Modern Intensity-Modulated Radiotherapy Techniques?
}

\author{
Vanessa Figlia $^{a} \quad$ Cristoforo Simonetto ${ }^{b}$ Markus Eidemüller ${ }^{b}$

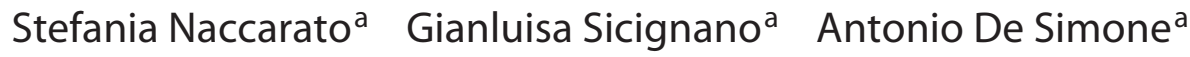 \\ Ruggero Ruggieria $^{\mathrm{a}}$ Rosario Mazzola ${ }^{\mathrm{a}}$ Christiane Matuschek ${ }^{c}$ Edwin Bölke $^{c}$ \\ Montserrat Pazos ${ }^{d}$ Maximilian Niyazi ${ }^{d}$ Claus Belka ${ }^{d}$ Filippo Alongi ${ }^{a}$ e \\ Stefanie Corradini ${ }^{d}$
}

\begin{abstract}
${ }^{a}$ Advanced Radiation Oncology Department, IRCCS Sacro Cuore Don Calabria Hospital, Negrar, Italy; ${ }^{\mathrm{b}}$ Institute of Radiation Medicine, Helmholtz Center Munich, Munich, Germany; ${ }^{C}$ Department of Radiation Oncology, Medical

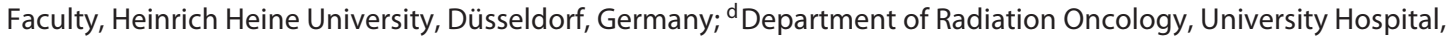

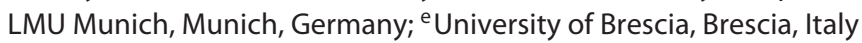

\section{Keywords}

Internal mammary chain - Regional nodal irradiation .

Breast cancer - Ischaemic heart disease - Secondary cancer

\begin{abstract}
Introduction: The aim of the present study was to estimate the impact of the addition of internal mammary chain (IMC) irradiation in node-positive left-sided breast cancer (BC) patients undergoing regional nodal irradiation (RNI) and comparatively evaluate excess relative and absolute risks of radiation-induced lung cancer/BC and ischaemic heart disease for intensity-modulated radiotherapy (IMRT) versus 3D conformal radiotherapy (3D-CRT). Methods: Four treatment plans were created (3D-CRT and IMRT -/+ IMC) for each of the 10 evaluated patients, and estimates of excess relative risk (ERR) and 10-year excess absolute risk (EAR) were calculated for radiation-induced lung cancer/BC and coronary events using linear, linear-exponential and plateau models. Results: The addition of IMC irradiation to RNI significantly increased the dose exposure of the heart, lung and contralateral breast using both techniques, increasing ERR for secondary lung cancer (58 vs. $44 \%, p=0.002$ ), contralateral BC
\end{abstract}

(49 vs. $31 \%, p=0.002$ ) and ischaemic heart disease (41 vs. $27 \%, p=0.002$, IMRT plans). IMRT significantly reduced the mean cardiac dose and mean lung dose as compared to 3DCRT, decreasing ERR for major coronary events (64\% 3D-CRT vs. $41 \%$ IMRT, $p=0.002$ ) and ERR for secondary lung cancer (75 vs. $58 \%, p=0.004)$ in IMC irradiation, without a significant impact on secondary contralateral BC risks. Conclusion: Although IMC irradiation has been shown to increase survival rates in node-positive $B C$ patients, it increased dose exposure of organs at risk in left-sided $B C$, resulting in significantly increased risks for secondary lung cancer/contralateral BC and ischaemic heart disease. In this setting, the adoption of IMRT seems advantageous when compared to 3D-CRT.

(c) 2020 S. Karger AG, Basel

\section{Introduction}

Currently, there is an ongoing debate about the role of internal mammary chain (IMC) irradiation in left-sided early breast cancer (BC) treatment $[1,2]$, as the additional morbidity caused by IMC irradiation contrasts with the actual advantage of this treatment in terms of overall sur- 
Fig. 1. Representation of 3D-CRT versus IMRT treatment beams in IMC plan.

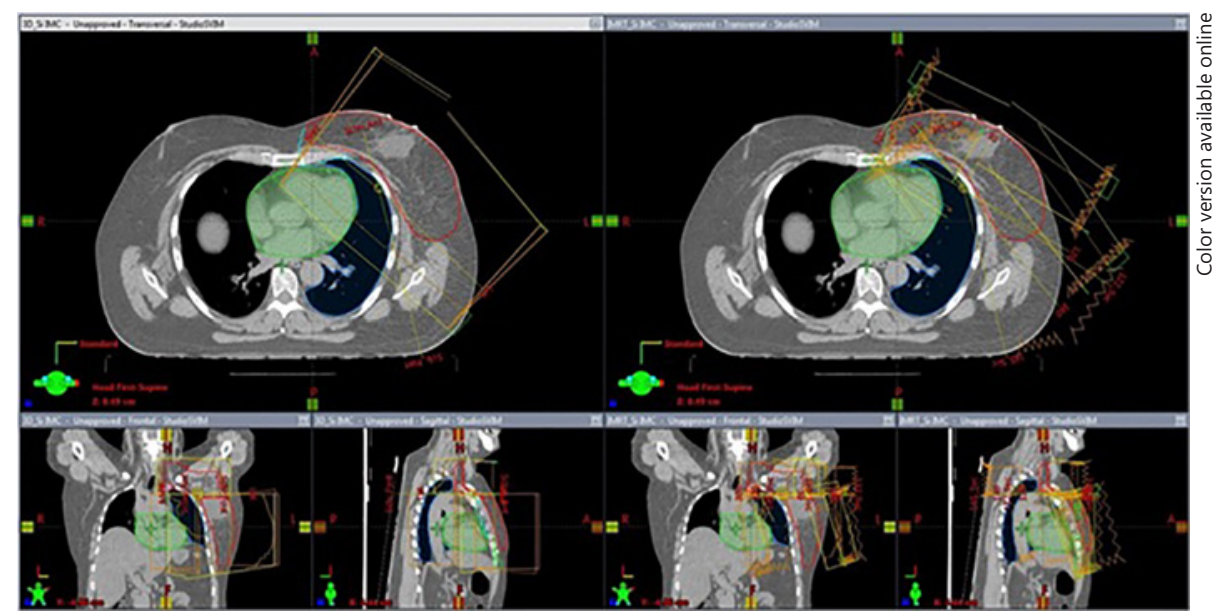

vival (OS), disease-free survival (DFS) and distant metastasis-free survival (DMFS). Randomised trials have reported a BC-specific survival benefit after regional nodal irradiation (RNI), especially when the IMC is treated, without an increase in cardiac morbidity and mortality [3-6]. As known from other studies, IMC irradiation can be associated with increased rates of cardiovascular disease and ischaemic heart disease, which remained increased for at least 20 years after treatment [7]. Due to the increased cardiovascular morbidity in left-sided BC radiotherapy, in the Danish Breast Cancer Cooperative Group Radiotherapy (DBCG)-IMN trial only patients with right-sided $\mathrm{BC}$ received internal mammary node radiotherapy, but organs-at-risk dose estimates were compared in right-sided versus left-sided BC patients. The study showed an absolute increase of $3 \%$ in OS when the IMC was added to RNI at a 7-year median follow-up. In fact, if a comparable gain is also assumed for left-sided patients, the benefits from IMC radiotherapy would outweigh the limitations of ischaemic heart morbidity [6]. Modern BC radiotherapy techniques, such as intensitymodulated radiotherapy (IMRT) or volumetric-modulated arc radiotherapy (VMAT), are able to minimize the high-dose exposure of the heart in left-sided BC [8-13] but only at the cost of an increased low-dose exposure, when compared to $3 \mathrm{D}$ conformal radiotherapy (3DCRT). This could potentially increase the risk for radiation-induced lung cancer and contralateral BC $[12,14]$. The IMC represents a complex target volume due to its close proximity to the heart. In this special case, the application of IMRT or VMAT could improve target volume coverage and at the same time spare cardiac dose exposure. The aim of the present study was to evaluate the impact of the addition of IMC irradiation in women with node-positive left $\mathrm{BC}$, candidates for adjuvant RNI. The study comparatively evaluates the excess relative and absolute risks of radiation-induced secondary lung cancer and $\mathrm{BC}$ and ischaemic heart disease for different modern radiotherapy techniques (IMRT vs. 3D-CRT).

\section{Materials and Methods}

\section{Patient Datasets and Treatment Planning}

For this risk modelling study, 40 treatment plans were generated using computed tomography datasets of 10 women with node-positive left-sided BC. All patients were previously treated using external beam radiotherapy following breast-conserving surgery in clinical routine. After re-contouring, 4 different treatment plans were generated for each patient with a prescription dose (Dp) of 50 Gy in 25 fractions: for both techniques (3D-CRT and IMRT), 2 treatment plans were created, based on the inclusion of IMC. Target volume delineation was performed according to international guidelines [15-17]. Furthermore, the IMC target volume was contoured around the internal thoracic artery/vein with a 5-mm margin from the caudal limit of level IV to the first 3 intercostal spaces [17]. To avoid any inter-observer variability, 1 physician delineated all organs at risk and target volumes in all datasets. The 3D-CRT technique consisted of 4-7 opposing wedged fields with 6/15 MV photons. Gantry angles were chosen in order to provide full target coverage and minimize dose exposure to the contralateral breast, ipsilateral lung and heart. IMRT plans consisted of 7-9 beams with $6 \mathrm{MV}$ photons for a Varian Trilogy Linac $\left({ }^{\circledR}\right.$ Varian Medical Systems, USA). Figure 1 shows the arrangement of the treatment beams for the 2 different techniques. In the present study, a respiratory gating/deep-inspiration breath hold (DIBH) technique was not included. Several studies have confirmed the substantial impact of DIBH on dosimetric endpoints, such as mean heart or ipsilateral lung dose in left-sided BC $[14,18,19]$. Nevertheless, even though in some countries DIBH is already routinely applied, it cannot be extensively used in most other countries. Therefore, the aim of the present study was to compare a 3D-CRT technique to IMRT - which is widely available. The authors are advocates of heart-sparing BC radiotherapy techniques and recommend to routinely use them. Therefore, the impact of an additionally applied respiratory gating will be evaluated in a further study.

All 3D-CRT and IMRT treatment plans were calculated using the AAA algorithm on an Eclipse ${ }^{\circledR}$ Treatment Planning System (Varian Medical Systems). Planning objectives during the forward (3D-CRT) and the inverse (IMRT) optimization process were full 
Table 1. Ten-year baseline risk values used for EAR calculations

\begin{tabular}{|c|c|c|c|c|}
\hline Age & Risk profile & Major coronary events ${ }^{\mathrm{a}}$ & Lung cancer ${ }^{\mathrm{b}}$ & Breast cancer ${ }^{c}$ \\
\hline 40 years & Intermediate risk & & & $0.8 \%$ \\
\hline 50 years & $\begin{array}{l}\text { Intermediate risk } \\
\text { High risk }\end{array}$ & $\begin{array}{l}0.5 \% \\
6.0 \%\end{array}$ & $\begin{array}{l}0.3 \% \\
2.2 \%\end{array}$ & \\
\hline 70 years & $\begin{array}{l}\text { Intermediate risk } \\
\text { High risk }\end{array}$ & $\begin{array}{l}2.8 \% \\
26.0 \%\end{array}$ & $\begin{array}{l}3.7 \% \\
15.0 \%\end{array}$ & \\
\hline
\end{tabular}

\begin{abstract}
a Baseline cardiac risks were calculated based on the standard Reynolds risk score algorithm [37] after subtracting the risk for stroke. "Intermediate-risk" profiles were estimated using the factors: systolic blood pressure, $125 \mathrm{~mm} \mathrm{Hg}$; total cholesterol, $208 \mathrm{mg} / \mathrm{dL}$; high-density lipoprotein, $52 \mathrm{mg} / \mathrm{dL}$; high-sensitivity C-reactive protein, $2.0 \mathrm{mg} / \mathrm{L}$; non-smoker; with no family history of myocardial infarction before the age of 60 years. "Highrisk" baseline risks were estimated using: systolic blood pressure, $135 \mathrm{~mm} \mathrm{Hg}$; total cholesterol, $235 \mathrm{mg} / \mathrm{dL}$; highdensity lipoprotein, $43 \mathrm{mg} / \mathrm{dL}$; high-sensitivity C-reactive protein, $4.3 \mathrm{mg} / \mathrm{L}$; smoker; with a family history of myocardial infarction before the age of 60 years. ${ }^{b}$ Baseline risks for lung cancer were estimated using the risk prediction formula from Bach et al. [25]. Only smokers were taken into account: the "intermediate-risk" profile corresponds to a woman who had smoked 10 cigarettes per day but quitted 5 years ago; "high-risk" corresponds to a current and continuing smoker with 30 cigarettes per day. In both cases, it was assumed that the woman started smoking at an age of 20 years. ${ }^{c}$ Baseline risks for contralateral breast cancer were estimated with the Tyrer-Cuzick model [29], assuming a 40-year-old woman with a weight of $65 \mathrm{~kg}$, height of $1.60 \mathrm{~m}$, menarche at age 14 years, a child birth at age 30 years and no known genetic predisposition or positive family history for breast cancer.
\end{abstract}

target coverage, defined as the volume that received at least $95 \%$ of the prescription dose $(\mathrm{V} 95 \% \mathrm{Dp}) \geq 95 \%$ planning target volume (PTV) and the dose received by $2 \%$ volumes of PTV (D2\%PTV) $\leq 107 \%$ of the prescription dose $(\mathrm{Dp})$ and adherence to dose constraints of organs at risk.

Referring to the QUANTEC recommendations for breast radiation therapy with conventional fractionation, we used the following dose constraints for the ipsilateral lung: V5 $<40 \%$, V20 $\leq 15 \%$, V $30 \leq 10 \%$, mean lung dose (MLD) $\leq 8-9$ Gy. For the mean heart dose, we used constraints derived from the study of Taylor et al. [20], which reported a mean heart dose of 6-7.3 Gy for leftsided BC, accepting higher doses when IMC irradiation was added [6]. For the contralateral breast, we tried to minimize the dose as much as possible, while keeping the mean contralateral breast dose $<3 \mathrm{~Gy}$.

\section{Estimates of Excess Relative Risk and Excess Absolute Risk}

Excess relative risk (ERR) and excess absolute risk (EAR) were calculated as described elsewhere [14]. The ERR was calculated as follows: $E R R=\delta O E D_{\text {linear }}$. Regarding the heart, the organ equivalent dose (OED) $O E D_{\text {linear }}$ corresponded to the mean cardiac dose $(\mathrm{MCD})$, and the linear risk coefficient $\delta_{\text {Darby }}=0.074 \mathrm{~Gy}^{-1}$, was taken from the dose-response relationship reported by Darby et al. [21], which relates to the risk of major coronary events (myocardial infarction, coronary revascularization, or death from ischaemic heart disease). For risk estimates of secondary lung cancer, $O E D_{\text {linear }}$ corresponded to the MLD and the parameter $\delta_{\text {Grantzau }}$ was derived from Grantzau et al. [22], who reported an ERR of $8.5 \%$ (95\% CI 3.1-23.3\%) per Gy of radiation-induced secondary lung cancer in 23,627 women following breast radiotherapy. Contralateral breast estimates were based on the $O E D_{\text {linear }}$, corresponding to the mean dose to the contralateral breast and the risk parameter $\delta_{\text {Stovall }}=0.20 \mathrm{~Gy}^{-1}$. This value was derived from BC patients treated before an age of 45 years [23].

The 10-year EAR was determined by multiplication of the ERR with the baseline risk: EAR =ERR BaselineRisk. The EAR was cal- culated for 2 different ages of treatment (50 and 70 years) and for 2 different risk profiles ("intermediate-risk"/"high-risk"), respectively. For the definition of the risk profiles and baseline risk calculation (Table 1), we followed Brenner et al. [24] for major coronary events and the risk prediction formula from Bach et al. [25] for baseline risks of lung cancer. Regarding secondary contralateral BC, radiation-induced secondary BC is assumed to be mainly relevant for exposure at younger ages [26-28]. Therefore, an intermediate 10-year baseline risk was estimated for a woman aged 40 years using the Tyrer-Cuzick model [29].

\section{Alternative Dose-Response Relationships}

Studies on secondary cancer risk after low-dose radiation exposure, and in particular the study on atomic bomb survivors, typically observe higher relative risks as compared to studies on secondary cancer. This can be explained by a non-linear dose-response relationship. Therefore, Schneider et al. [30] have introduced the concept of OED. The OED is assumed to be proportional to the organ cancer risk but depends only on the dose distribution within the organ itself. Thus, the OED is suitable to compare the potential harm of different dose distributions within the same person. However, it should be noted that the coefficients $\delta_{\text {Grantzau }}$ and $\delta_{\text {Stovall }}$ do not apply for risk calculation with non-linear OEDs. Plausible functional forms of dose-response relationships were derived mechanistically and adjusted to the risks observed in atomic bomb survivors and patients with Hodgkin's disease [31]. For comparison, we calculated OED using linear-exponential $\left(O E D_{\text {linear-exp }}\right)$ and plateau dose-response $\left(O E D_{\text {plateau }}\right)$ models as described elsewhere [15]. The organ-specific model parameters used for lung cancer where $\alpha=0.022 \mathrm{~Gy}^{-1}$ and $\beta=0.056 \mathrm{~Gy}^{-1}$, while for the contralateral breast the parameters $\alpha=0.041 \mathrm{~Gy}^{-1}$ and $\beta=0.115 \mathrm{~Gy}^{-1}$ were used.

\section{Statistical Analysis}

Statistical analyses were conducted using Matlab R2018b (MathWorks, USA). Wilcoxon signed-rank tests were used to es- 


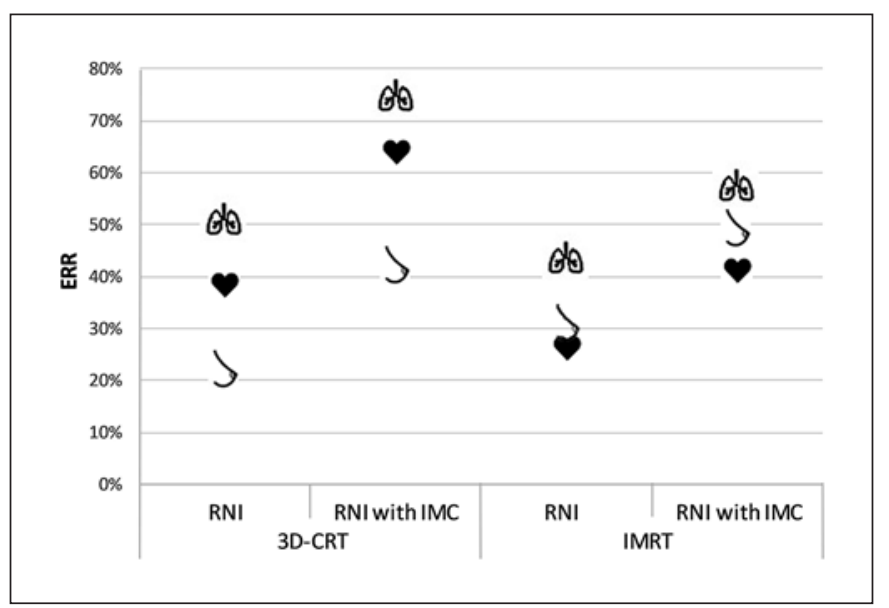

Fig. 2. Estimates of excess relative risk (ERR) for radiation-induced secondary lung and contralateral breast cancer and ischaemic heart disease for the different treatment modalities and target volumes (-/+IMC).

timate statistical significance of differences between plans. All data were recorded with means and standard deviation, and $p$ values $<0.05$ were considered statistically significant.

\section{Results}

\section{Heart Exposure and Risk Estimation for Major \\ Coronary Events}

An overview of the OEDs of the different treatment modalities is tabulated in Table 2. The largest dose variations were seen for the MCD in 3D-CRT plans. The addition of the IMC to the target volume significantly increased the mean MCD using both treatment techniques, 3D-CRT and IMRT: in 3D-CRT plans, the MCD was 5.18 \pm 2.26 Gy versus $8.68 \pm 2.72 \mathrm{~Gy}(-/+\mathrm{IMC}$ irradiation, $p=$ $0.002)$; in IMRT plans, it was $3.59 \pm 0.97$ Gy versus $5.57 \pm$ 1.58 Gy $(-/+$ IMC, $p=0.002)$. As shown in Table 2, the lowest MCD was reached in IMRT plans without addition of the IMC. As compared to 3D-CRT, the use of IMRT significantly reduced the MCD in both cases, in RNI only $(p=0.02)$ and when the IMC was included in the target volume $(p=0.002)$. Regarding the volume of the heart receiving $5 \mathrm{~Gy}$ (V5Gy), the volume receiving at least $5 \mathrm{~Gy}$ was significantly larger when IMC was added to the target volume (3D-CRT: $15.79 \pm 7.38 \%$ vs. $27.62 \pm$ $8.72 \%$; $-/+$ IMC irradiation, $p=0.002$; in IMRT plans: $15.14 \pm 7.65 \%$ vs. $31.05 \pm 13.20 \%$; $-/+$ IMC, $p=0.002$ ). Nevertheless, there was no significant difference between IMC irradiation using 3D-CRT or IMRT $(p=0.2)$.

An overview of estimated ERR and 10-year EAR for ischaemic heart disease for the different treatment modalities and typical baseline risks is shown in Figures 2-4. The ERR for major coronary events was 38\% in 3D-CRT

कำลำ +1 $+\begin{gathered}\infty \\ +1+1\end{gathered}$ 즌 ம் ते 
Fig. 3. Estimates of 10-year excess absolute risk (EAR) for radiation-induced secondary lung cancer and ischaemic heart disease for treatment age of 50 years. The Figure shows values for different treatment modalities, target volumes $(-/+\mathrm{IMC})$ and 2 different baseline risks (see Table 1).

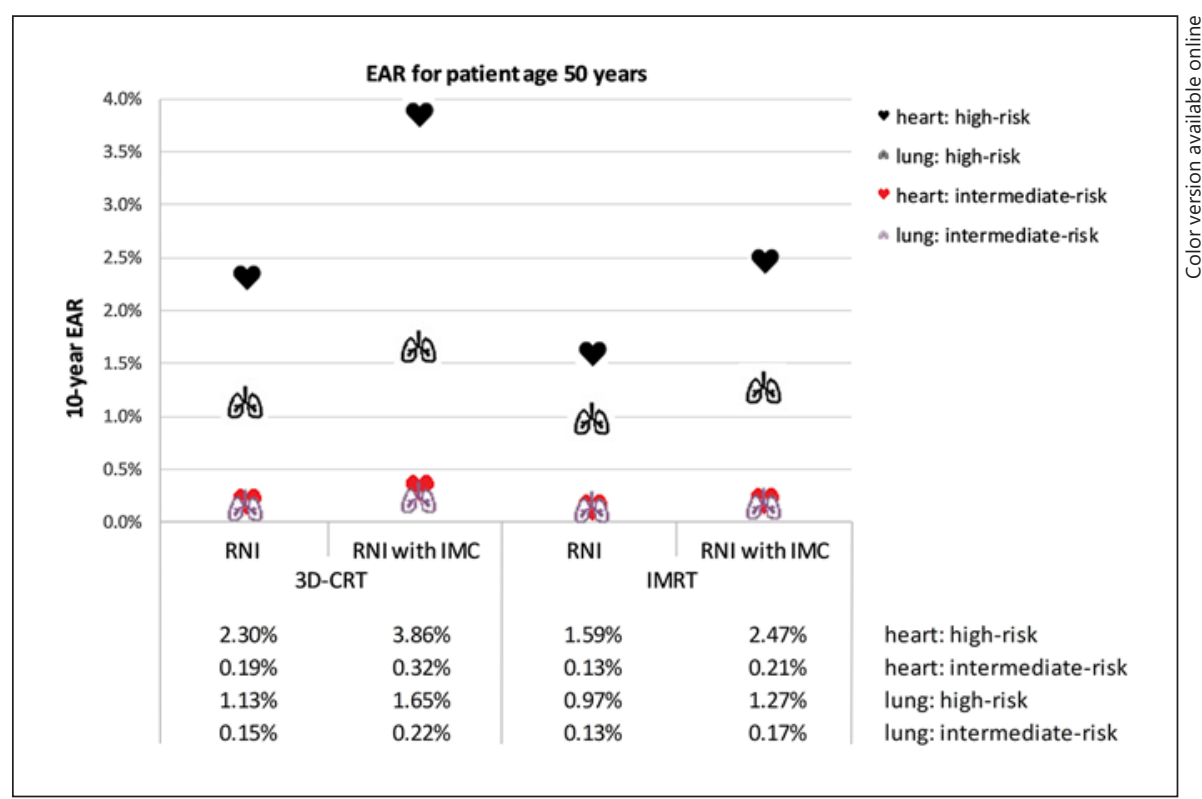

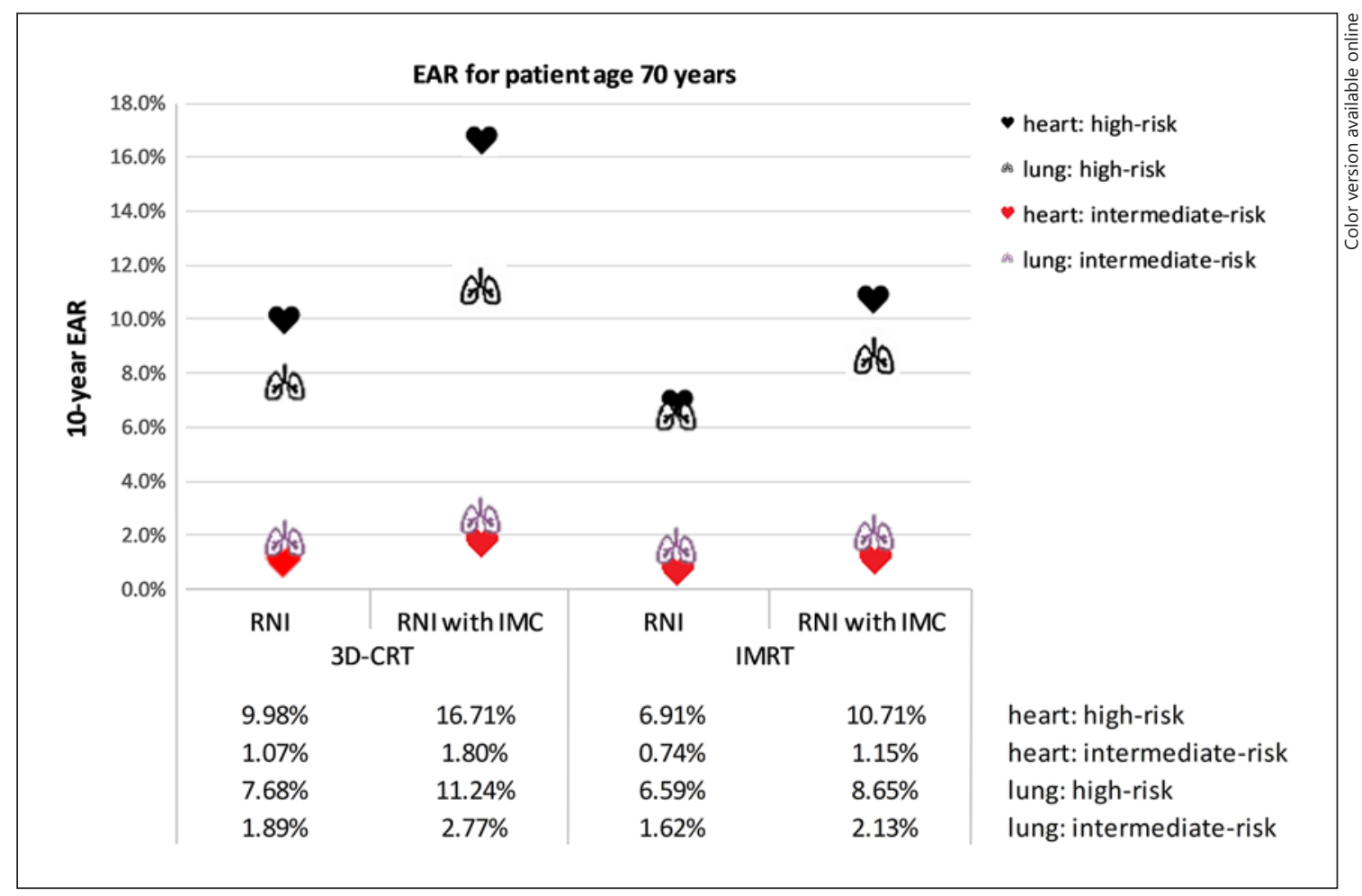

Fig. 4. Estimates of 10-year excess absolute risk (EAR) for radiation-induced secondary lung cancer and ischaemic heart disease for treatment age of 70 years. The Figure shows values for different treatment modalities, target volumes $(-/+\mathrm{IMC})$ and 2 different baseline risks (see Table 1$)$.

without IMC irradiation and 64\% for 3D-CRT with IMC irradiation. When the IMRT technique was used, ERR was $27 \%$ and $41 \%$ without and with IMC, respectively. Therefore, the mean predicted risk increase through IMC irradiation was less pronounced in IMRT plans. Accordingly, also a higher 10-year EAR for major coronary events was shown for 3D-CRT as compared to IMRT plans. Ten-year EAR calculations for major coronary events were strongly influenced by cardiac baseline risks (Table 1 ). Elderly patients (70 years) presenting with relevant cardiovascular disease risk factors ("high-risk"), had an absolute 10-year risk reduction of 3\% for isch- 
Fig. 5. 3D-CRT versus IMRT dosimetric comparison in non-IMC plans. Square: 3D-CRT plan; triangle: IMRT plan; red: PTV; blue: ipsilateral lung; green: heart; orange: contralateral breast.

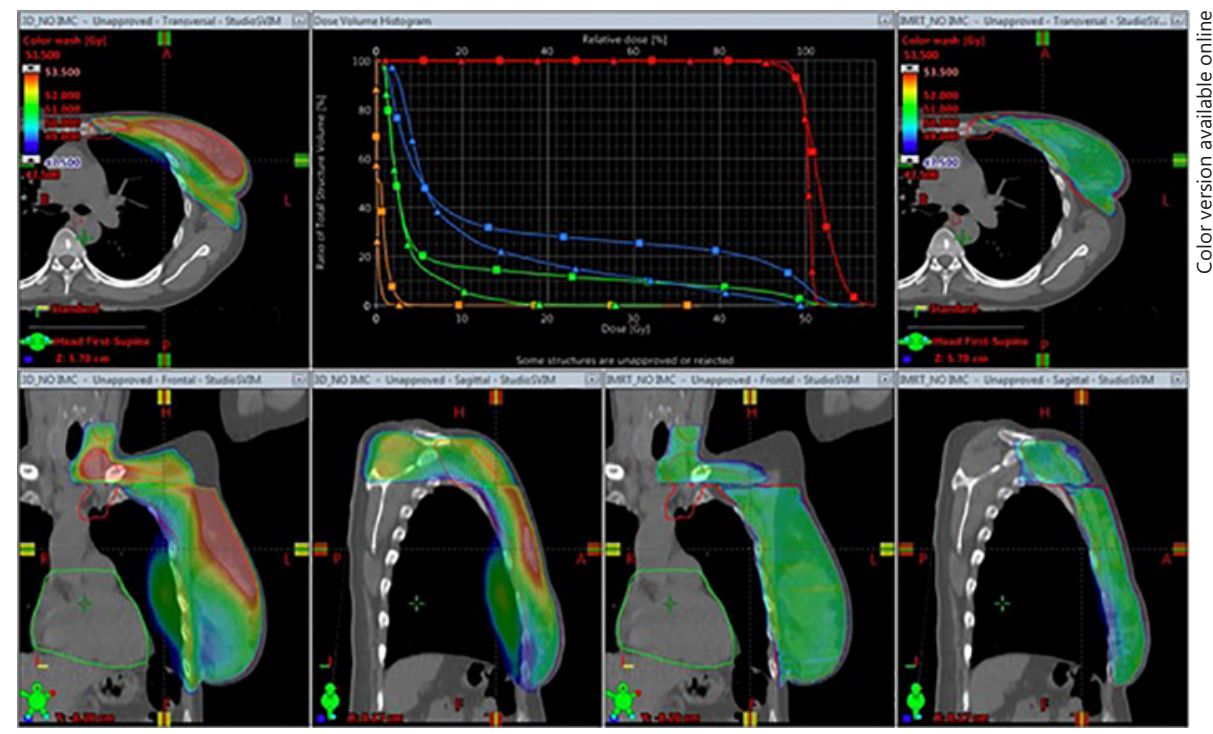

aemic heart disease using an IMRT technique rather than 3D-CRT (-IMC). In case of IMC irradiation, the use of IMRT was associated with an overall 10-year cardiac EAR reduction of $6 \%$ compared to 3D-CRT. Nevertheless, even if using the IMRT technique, the estimated additional cardiac 10-year risk from irradiating the IMC was large (4\%), thus compromising the benefit for patients with high cardiovascular risk (Fig. 4).

\section{Lung Exposure and Risk Estimation for Secondary \\ Lung Cancer}

IMC irradiation also significantly increased the MLD in 3D-CRT and IMRT plans ( $p=0.002$, respectively; Table 2). The mean MLD value was $6.02 \pm 0.79$ Gy in $3 \mathrm{D}$-CRT plans without IMC irradiation and $8.81 \pm 1.45$ Gy in 3D-CRT plans including the IMC. Regarding IMRT plans, the MLD was $5.17 \pm 0.87 \mathrm{~Gy}(-\mathrm{IMC})$ and $6.78 \pm 0.91$ Gy (+IMC), respectively. However, dose volumes receiving more than 5 Gy were significantly higher using an IMRT technique as compared to 3DCRT, irrespective of the inclusion of the IMC: $31 \pm 11 \%$ versus $21 \pm 2 \%$ without IMC $(p=0.002)$ and $35 \pm 12 \%$ versus $25 \pm 3 \%$ with IMC ( $p=0.03)$. As a result, while IMRT significantly reduced MLD, the difference in non-linear OEDs was not statistically significant using the 3D-CRT technique.

Figure 2 shows the ERR for radiation-induced secondary lung cancer for each treatment modality estimated using the linear OED model. The ERR for secondary lung cancer was significantly influenced by IMC irradiation in both 3D-CRT and IMRT plans $(p=0.002)$. We estimated a reduction in the ERR for secondary lung cancer risk when using the IMRT technique in plans with RNI only ( 51 vs. $44 \%, p=0.04$ ) and when the IMC was added to RNI (75 vs. $58 \%, p=0.004)$. Corresponding exemplary 10-year EAR calculations are depicted in Figures 3 and 4 for patients aged 50 or 70 years at treatment, respectively. However, it is important to note that the above-mentioned OED calculations have shown that the advantage of the IMRT technique may disappear when the doseresponse relationship is not linear. Moreover, the applied baseline risk for lung cancer (Table 1) and the smoking status had the strongest impact on 10-year EAR estimation: intermediate-risk patients showed substantially lower 10-year EAR values for secondary lung cancer as compared to high-risk patients.

\section{Exposure and Secondary Cancer Risk Estimation for the Contralateral Breast}

Mean dose of the contralateral breast was $1.10 \pm 1.21$ Gy in 3D-CRT without IMC irradiation and $2.11 \pm 2.20$ Gy for 3D-CRT plans with IMC as compared to $1.55 \pm$ $1.71 \mathrm{~Gy}$ for IMRT plans without IMC and $2.47 \pm 2.10 \mathrm{~Gy}$ for IMRT plans with IMC, respectively (Table 2). The inclusion of the IMC in the target volume significantly increased the mean dose and non-linear OEDs of the contralateral breast in 3D-CRT and IMRT plans $(p=0.002$, respectively). In this case, the use of an IMRT technique had no significant impact to reduce contralateral breast mean dose $(p>0.5)$.

We applied the linear model to estimate the ERR for radiation-induced contralateral $\mathrm{BC}$ for each treatment modality, as shown in Figure 2. The ERR for secondary $\mathrm{BC}$ was significantly increased by IMC irradiation in 3DCRT and IMRT plans $(p=0.002)$ but, as stated before, there was no significant difference for the IMRT versus 3D-CRT technique: neither in -IMC plans (31 vs. $22 \%$, $p=0.56$ ), nor in +IMC plans ( 49 vs. $42 \%, p=1$ ).

Estimated intermediate-risk 10-year EARs at age 40 years were $0.35 \%$ in $3 \mathrm{D}$-CRT -IMC and $0.67 \%+$ IMC ir- 
Fig. 6. 3D-CRT versus IMRT dosimetric comparison in IMC plans. Square: 3DCRT plan; triangle: IMRT plan; red: PTV; blue: ipsilateral lung; green: heart; orange: contralateral breast.

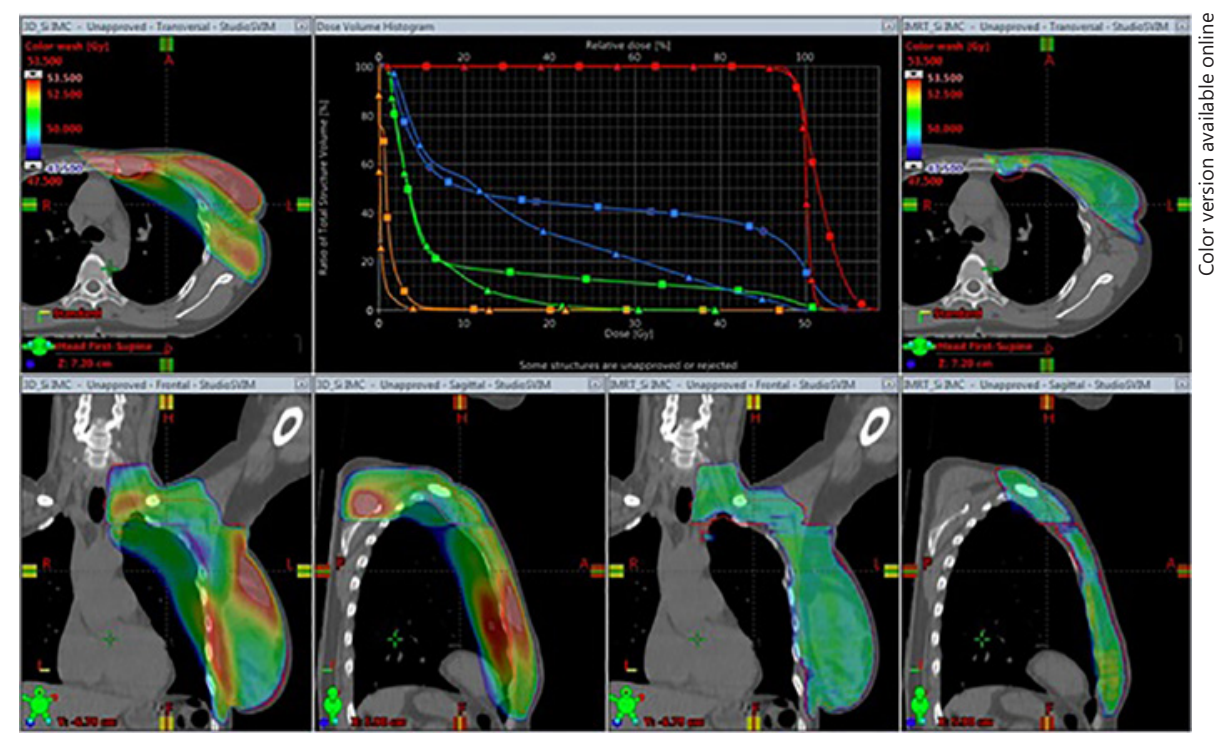

radiation, and $0.50 \%$ in IMRT $-\mathrm{IMC}$ and $0.79 \%+\mathrm{IMC}$ irradiation. Figures 5 and 6 show 3D-CRT versus IMRT dosimetric comparison in non-IMC and IMC plans.

\section{Discussion/Conclusion}

The addition of RNI in node-positive $\mathrm{BC}$ or high-risk node-negative $\mathrm{BC}$ has recently shown to increase DMFS, which translates into better DFS [32] or even OS [31]. This has recently also been confirmed by the EBCTCG meta-analysis presented in San Antonio in 2018 (data not yet published). However, the debate about the IMC irradiation in left-sided BC treatment is still open for discussion, as the additional morbidity caused by IMC irradiation contrasts with its actual advantage. The $\mathrm{BC}$-specific survival benefit after RNI including the IMC has been reported by several randomized trials. In 2015, Budach et al. [33] published a large meta-analysis of the 3 latest trials showing that additional regional radiotherapy, including the internal mammary and medial supraclavicular lymph nodes, in node-positive $\mathrm{BC}$ or high-risk node-negative $\mathrm{BC}$, statistically significantly improved DFS, DMFS and OS in stage I-III BC. Later, the same group focused on the individual impact of the different axillary target volumes. The network meta-analysis showed that the improvement of cancer-specific survival through RNI is mainly achieved by inclusion of the IMC, deriving from a reduction in DMFS [34].

The clinical attention to radiation-induced heart damage and secondary cancer has recently increased. In view of the continuously increasing long-term survival rates of $\mathrm{BC}$ patients, one of the main goals in radiotherapy is the attempt to reduce radiation-induced morbidity. Especially in the context of multimodal treatment protocols, the synergistic effects of radiotherapy and cardiotoxic anthracycline-based systemic therapy or targeted therapies (anti-Her2) are relevant $[35,36]$, as they are also associated with an increase in cardiomyopathy and heart failure $[37,38]$. In this scenario, it seems reasonable to reduce the cardiac dose exposure by applying modern technological developments. Regarding radiation-induced cardiac morbidity, in 2013, Darby et al. [21] found a relative risk for major coronary events of 7.4\% (95\% CI 2.9-14.5) per every $1 \mathrm{~Gy}$ increase in MCD. As a result, new alternative treatment techniques, such as respiratory-gated radiotherapy using the DIBH technique or IMRT-VMAT techniques, have been introduced into clinical practice to reduce heart and lung exposure $[18,39,40]$. In a recent risk modelling study, Simonetto et al. [19] showed that heartsparing techniques should be offered to all patients who have high cardiac risks at baseline, a high dose exposure of the heart, or a favourable tumour prognosis, regardless of their age at diagnosis. A large population-based study of 29,102 BC patients supports these findings [41]. In fact, elderly women with a history of cardiac disease had increased rates of coronary interventions and a survival reduction following breast radiotherapy. The present study showed a positive impact of the adoption of modern intensity-modulated radiation techniques when treating RNI including the IMC and is in line with other studies [42]. The use of IMRT resulted in an MCD reduction and consequently ERR and 10-year EAR reduction for ischaemic heart disease. An analysis of the 10-year EAR for major coronary events revealed that IMC irradiation significantly increased the risk for cardiovascular disease in 3D-CRT and IMRT plans as compared to RNI alone, but the mean predicted risk increase through IMC irradiation was less pronounced in IMRT plans. Nevertheless, even if using the IMRT technique, the estimated ad- 
ditional cardiac risk from irradiating the IMC was large (up to $4 \%$ ), thus compromising the benefit for patients with high cardiovascular risk (Fig. 4).

Similarly, the risk of radiation-induced secondary lung cancer in the current study was mainly influenced by the radiation technique (3D-CRT vs. IMRT). Many population-based studies have confirmed the risk of radiationinduced lung cancer [43-49], but comparative studies estimating radiation-induced lung carcinogenesis following different breast radiotherapy techniques are rare [14, 44]. Most of them compared a 3D-CRT approach with other modern techniques in the setting of breast radiotherapy only, without inclusion of RNI. In contrast to these reports, the present study evaluated the dosimetric impact of modern $\mathrm{BC}$ radiotherapy within the context of RNI and especially focused on the addition of the IMC region. As RNI and the IMC represent complex target volumes, the application of IMRT could spare dose exposure of organs at risk. Both scenarios (RNI -/+ IMC) showed lower absolute risks for secondary lung cancer in IMRT plans. In this context, it has to be mentioned that the risk of radiation-induced lung cancer is highly influenced by the smoking status. Usually, non-smoking women undergoing radiotherapy have no higher risk for radiation-induced lung cancer [48]. It has been shown that ever-smoking was an independent risk factor for secondary lung cancer, while radiotherapy alone was not [49]. Taking into account all of the above-mentioned aspects, smoking cessation might have a higher impact on secondary lung cancer reduction than the influence of different radiotherapy techniques. In our study, the risk of radiation-induced contralateral secondary BC was similarly significantly influenced by IMC irradiation but not by the treatment technique itself. Other dosimetric studies showed the lowest mean contralateral breast dose using 3D conformal tangential and volumetric modulated arc techniques, whereas conventional IMRT techniques have resulted in an increase in contralateral breast dose up to $30 \%$ [50]. The present study showed no statistically significant difference in mean dose to the contralateral breast in IMRT plans as compared to 3D-CRT. Consequently, the 10-year predicted EAR for secondary contralateral $\mathrm{BC}$ was only significantly influenced by the addition of IMC irradiation. It is important to note that the actual risk of radiation-induced contralateral secondary $\mathrm{BC}$ might probably be lower than the predicted one [51].

The present study has some limitations due to the small sample size and the uncertainties of risk modelling. In fact, due to the small size of the study, the results may not be fully representative, as individual anatomy can have a significant impact on radiotherapy planning, although we excluded patients with unfavourable anatomy. In addition, a clear dose-response relationship for sec- ondary lung and contralateral $\mathrm{BC}$ after $\mathrm{BC}$ radiotherapy has not yet been established, and we thus applied the OED concept for a relative comparison of different treatment methods with regard to a specific organ cancer risk. For $\mathrm{BC}$, controversial evidence exists regarding the dependence on age at exposure. For example, in the atomic bomb survivors of Hiroshima and Nagasaki, the ERR was found to depend only slightly on exposure age after correcting for attained age $[52,53]$. Furthermore, regarding cardiovascular disease risk prediction, the linear dose-response relationship has long been debated. Different biological radiation effects seem to be responsible for cardiovascular detriment at low doses and differ from those at high doses [54-56]. It has been suggested that a low-dose exposure to the left ventricle (volume receiving $5 \mathrm{~Gy}$ ) might be a better predictor for acute coronary events than the mean heart dose [57]. We, therefore, added the V5Gy of the entire heart to give an estimate of low-dose exposure of the heart. Nevertheless, there was no significant difference between 3D-CRT and IMRT in IMC irradiation regarding the V5Gy of the heart.

In conclusion, IMC irradiation has been shown to increase survival rates in women with node-positive BC. Nevertheless, the addition of IMC to RNI in left-sided BC also increases heart, lung and contralateral breast dose exposure and can result in a significantly increased risk for secondary lung cancer and contralateral $\mathrm{BC}$ and ischaemic heart disease. The present study showed that the adoption of IMRT seems advantageous in this setting to mitigate cardiac toxicity and the risk of radiation-induced secondary cancers.

\section{Statement of Ethics}

Ethics committee approval was not required since this was not a clinical study performed on patients but a dosimetric simulation study. Informed consent was not required since the dosimetric simulation study was performed on anonymous patient data.

\section{Conflict of Interest Statement}

The authors have no conflicts of interest to declare.

\section{Funding Sources}

The authors received no specific funding for this work.

\section{Author Contributions}

V.F., F.A., C.S., S.C. and R.R. wrote the manuscript and tables and organized the study. S.N., G.S., A.D.S. and R.R. planned treatment of the patients. C.S. performed the statistical analysis. All authors read and approved the final manuscript. 


\section{References}

1 Haffty BG, Whelan T, Poortmans PM. Radiation of the internal mammary nodes: is there a benefit? J Clin Oncol. 2016 Feb;34(4):297-9.

2 Whelan TJ, Olivotto IA, Parulekar WR, Ackerman I, Chua BH, Nabid A, et al.; MA.20 Study Investigators. Regional nodal irradiation in early-stage breast cancer. N Engl J Med. 2015 Jul;373(4):307-16.

3 Matzinger O, Heimsoth I, Poortmans P, Collette L, Struikmans H, Van Den Bogaert W, et al.; EORTC Radiation Oncology \& Breast Cancer Groups. Toxicity at three years with and without irradiation of the internal mammary and medial supraclavicular lymph node chain in stage I to III breast cancer (EORTC trial 22922/10925). Acta Oncol. 2010;49(1): 24-34.

4 Haussmann J, Budach W, Tamaskovics B, Bölke E, Corradini S, Djiepmo-Njanang FJ, et al. Which target volume should be considered when irradiating the regional nodes in breast cancer? Results of a network-meta-analysis. Radiat Oncol. 2019 Jun;14(1):102.

5 Harris EE, Correa C, Hwang WT, Liao J, Litt HI, Ferrari VA, et al. Late cardiac mortality and morbidity in early-stage breast cancer patients after breast-conservation treatment. J Clin Oncol. 2006 Sep;24(25):4100-6.

6 Thorsen LB, Thomsen MS, Berg M, Jensen I, Josipovic M, Overgaard M, et al; Danish Breast Cancer Cooperative Group Radiotherapy committee. CT-planned internal mammary node radiotherapy in the DBCG-IMN study: benefit versus potentially harmful effects. Acta Oncol. 2014 Aug;53(8):1027-34.

7 Henson KE, McGale P, Taylor C, Darby SC. Radiation-related mortality from heart disease and lung cancer more than 20 years after radiotherapy for breast cancer. Br J Cancer. 2013 Jan;108(1):179-82.

8 Mast ME, Heijenbrok MW, van KempenHarteveld ML, Petoukhova AL, Scholten AN, Wolterbeek R, et al. Less increase of CT-based calcium scores of the coronary arteries: effect three years after breast-conserving radiotherapy using breath-hold. Strahlenther Onkol. 2016 Oct;192(10):696-704.

9 Jöst V, Kretschmer M, Sabatino M, Würschmidt F, Dahle J, Ueberle F, et al. Heart dose reduction in breast cancer treatment with simultaneous integrated boost: comparison of treatment planning and dosimetry for a novel hybrid technique and 3D-CRT. Strahlenther Onkol. 2015 Sep;191(9):734-41.

10 Fiorentino A, Ruggieri R, Giaj-Levra N, Sicignano G, Di Paola G, Naccarato S, et al. Three-dimensional conformal versus intensity modulated radiotherapy in breast cancer treatment: is necessary a medical reversal? Radiol Med (Torino). 2017 Feb;122(2):146-53.

11 Osman SO, Hol S, Poortmans PM, Essers M. Volumetric modulated arc therapy and breath-hold in image-guided locoregional left-sided breast irradiation. Radiother Oncol. 2014 Jul;112(1):17-22.

12 Abo-Madyan Y, Aziz MH, Aly MM, Schneider F, Sperk E, Clausen S, et al. Second cancer risk after 3D-CRT, IMRT and VMAT for breast cancer. Radiother Oncol. 2014 Mar; 110(3):471-6.
13 Dess RT, Liss AL, Griffith KA, Marsh RB, Moran JM, Mayo $\mathrm{C}$, et al. Ischemic cardiac events following treatment of the internal mammary nodal region using contemporary radiation planning techniques. Int J Radiat Oncol Biol Phys. 2017 Dec;99(5):1146-53.

14 Corradini S, Ballhausen $\mathrm{H}$, Weingandt $\mathrm{H}$, Freislederer P, Schönecker S, Niyazi M, et al. Left-sided breast cancer and risks of secondary lung cancer and ischemic heart disease: Effects of modern radiotherapy techniques. Strahlenther Onkol. 2018 Mar;194(3):196205

15 Offersen BV, Boersma LJ, Kirkove C, Hol S, Aznar MC, Biete Sola A, et al. ESTRO consensus guideline on target volume delineation for elective radiation therapy of early stage breast cancer. Radiother Oncol. 2015 Jan;114(1):310.

16 Feng M, Moran JM, Koelling T, Chughtai A, Chan JL, Freedman L, et al. Development and Validation of a Heart Atlas to Study Cardiac Exposure to Radiation Following Treatment for Breast Cancer. Int J Radiat Oncol Biol Phys. 2011 Jan;79(1):10-8.

17 Sautter-Bihl ML, Sedlmayer F, Budach W, Dunst J, Feyer P, Fietkau R, et al.; Breast Cancer Expert Panel of the German Society of Radiation Oncology (DEGRO). DEGRO practical guidelines: radiotherapy of breast cancer III-radiotherapy of the lymphatic pathways. Strahlenther Onkol. 2014 Apr;190(4):34251.

18 Duma MN, Baumann R, Budach W, Dunst J, Feyer P, Fietkau R, et al.; Breast Cancer Expert Panel of the German Society of Radiation Oncology (DEGRO). Heart-sparing radiotherapy techniques in breast cancer patients: a recommendation of the breast cancer expert panel of the German society of radiation oncology (DEGRO). Strahlenther Onkol. 2019 Oct;195(10):861-71.

19 Simonetto C, Eidemüller M, Gaasch A, Pazos M, Schönecker S, Reitz D, et al. Does deep inspiration breath-hold prolong life? Individual risk estimates of ischaemic heart disease after breast cancer radiotherapy. Radiother Oncol. 2019 Feb;131:202-7.

20 Taylor CW, Brønnum D, Darby SC, Gagliardi G, Hall P, Jensen MB, et al. Cardiac dose estimates from Danish and Swedish breast cancer radiotherapy during 1977-2001. Radiother Oncol. 2011 Aug;100(2):176-83.

21 Darby SC, Ewertz M, McGale P, Bennet AM, Blom-Goldman U, Brønnum D, et al. Risk of ischemic heart disease in women after radiotherapy for breast cancer. N Engl J Med. 2013 Mar;368(11):987-98.

22 Grantzau T, Thomsen MS, Væth M, Overgaard J. Risk of second primary lung cancer in women after radiotherapy for breast cancer. Radiother Oncol. 2014 Jun;111(3):366-73.

23 Stovall M, Smith SA, Langholz BM, Boice JD Jr, Shore RE, Andersson M, et al; Women's Environmental, Cancer, and Radiation Epidemiology Study Collaborative Group. Dose to the contralateral breast from radiotherapy and risk of second primary breast cancer in the WECARE study. Int J Radiat Oncol Biol Phys. 2008 Nov;72(4):1021-30.
24 Brenner DJ, Shuryak I, Jozsef G, Dewyngaert KJ, Formenti SC. Risk and risk reduction of major coronary events associated with contemporary breast radiotherapy. JAMA Intern Med. 2014 Jan;174(1):158-60.

25 Bach PB, Kattan MW, Thornquist MD, Kris MG, Tate RC, Barnett MJ, et al. Variations in lung cancer risk among smokers. J Natl Cancer Inst. 2003 Mar;95(6):470-8

26 Storm HH, Andersson M, Boice JD Jr, Blettner M, Stovall M, Mouridsen HT, et al. Adjuvant radiotherapy and risk of contralateral breast cancer. J Natl Cancer Inst. 1992 Aug;84(16): 1245-50.

27 Preston DL, Mattsson A, Holmberg E, Shore R, Hildreth NG, Boice JD Jr. Radiation effects on breast cancer risk: a pooled analysis of eight cohorts. Radiat Res. 2002 Aug;158(2): 220-35.

28 Schneider U, Walsh L. Age at exposure and attained age variations of cancer risk in the Japanese A-bomb and radiotherapy cohorts. Med Phys. 2015 Aug;42(8):4755-61.

29 IBIS (International Breast Cancer Intervention Study) Online Tyrer-Cuzick Model Breast Cancer Risk Evaluation Tool [Internet]. Available from: https://ibis.ikonopedia. com

30 Schneider U, Sumila M, Robotka J. Site-specific dose-response relationships for cancer induction from the combined Japanese Abomb and Hodgkin cohorts for doses relevant to radiotherapy. Theor Biol Med Model. 2011 Jul;8(1):27.

31 Hennequin C, Bossard N, Servagi-Vernat S, Maingon P, Dubois JB, Datchary J, et al. Tenyear survival results of a randomized trial of irradiation of internal mammary nodes after mastectomy. Int J Radiat Oncol Biol Phys. 2013 Aug;86(5):860-6.

32 Reed DR, Lindsley SK, Mann GN, AustinSeymour M, Korssjoen T, Anderson BO, et al. Axillary lymph node dose with tangential breast irradiation. Int J Radiat Oncol Biol Phys. 2005 Feb;61(2):358-64.

33 Budach W, Bölke E, Kammers K, Gerber PA, Nestle-Krämling C, Matuschek C. Adjuvant radiation therapy of regional lymph nodes in breast cancer - a meta-analysis of randomized trials - an update. Radiat Oncol. 2015 Dec;10: 258.

34 Thorsen LB, Offersen BV, Danø H, Berg M, Jensen I, Pedersen AN, et al. DBCG-IMN: A Population-Based Cohort Study on the Effect of Internal Mammary Node Irradiation in Early Node-Positive Breast Cancer. J Clin Oncol. 2016 Feb;34(4):314-20.

35 Perez EA, Romond EH, Suman VJ, Jeong JH, Davidson NE, Geyer CE Jr, et al. Four-year follow-up of trastuzumab plus adjuvant chemotherapy for operable human epidermal growth factor receptor 2-positive breast cancer: joint analysis of data from NCCTG N9831 and NSABP B-31. J Clin Oncol. 2011 Sep;29(25):3366-73. 
36 Romond EH, Jeong JH, Rastogi P, Swain SM, Geyer CE Jr, Ewer MS, et al. Seven-year follow-up assessment of cardiac function in NSABP B-31, a randomized trial comparing doxorubicin and cyclophosphamide followed by paclitaxel (ACP) with ACP plus trastuzumab as adjuvant therapy for patients with node-positive, human epidermal growth factor receptor 2-positive breast cancer. J Clin Oncol. 2012 Nov;30(31):3792-9.

37 Ridker PM, Buring JE, Rifai N, Cook NR. Development and validation of improved algorithms for the assessment of global cardiovascular risk in women: the Reynolds Risk Score. JAMA. 2007 Feb;297(6):611-9.

38 Chambless LE, Heiss G, Shahar E, Earp MJ, Toole J. Prediction of ischemic stroke risk in the Atherosclerosis Risk in Communities Study. Am J Epidemiol. 2004 Aug;160(3):259-69.

39 Schönecker S, Heinz C, Söhn M, Haimerl W, Corradini S, Pazos M, et al. Reduction of cardiac and coronary artery doses in irradiation of left-sided breast cancer during inspiration breath hold: A planning study. Strahlenther Onkol. 2016 Nov;192(11):750-8.

40 Nguyen MH, Lavilla M, Kim JN, Fang LC. Cardiac sparing characteristics of internal mammary chain radiotherapy using deep inspiration breath hold for left-sided breast cancer. Radiat Oncol. 2018 May;13(1):103.

41 Darby SC, McGale P, Taylor CW, Peto R. Long-term mortality from heart disease and lung cancer after radiotherapy for early breast cancer: prospective cohort study of about 300,000 women in US SEER cancer registries. Lancet Oncol. 2005 Aug;6(8):557-65.

42 Ranger A, Dunlop A, Hutchinson K, Convery $\mathrm{H}$, Maclennan MK, Chantler H, et al. A Dosimetric Comparison of Breast Radiotherapy Techniques to Treat Locoregional Lymph Nodes Including the Internal Mammary
Chain. Clin Oncol (R Coll Radiol). 2018 Jun; 30(6):346-53.

43 Kirova YM, De Rycke Y, Gambotti L, Pierga JY, Asselain B, Fourquet A; Institut Curie Breast Cancer Study Group. Second malignancies after breast cancer: the impact of different treatment modalities. Br J Cancer. 2008 Mar;98(5):870-4.

44 Prochazka M, Granath F, Ekbom A, Shields PG, Hall P. Lung cancer risks in women with previous breast cancer. Eur J Cancer. 2002 Jul; 38(11):1520-5.

45 Neugut AI, Murray T, Santos J, Amols H, Hayes MK, Flannery JT, et al. Increased risk of lung cancer after breast cancer radiation therapy in cigarette smokers. Cancer. 1994 Mar;73(6):1615-20

46 Inskip PD, Stovall M, Flannery JT. Lung cancer risk and radiation dose among women treated for breast cancer. J Natl Cancer Inst. 1994 Jul;86(13):983-8.

$47 \mathrm{Ng}$ J, Shuryak I, Xu Y, Clifford Chao KS, Brenner DJ, Burri RJ. Predicting the risk of secondary lung malignancies associated with whole-breast radiation therapy. Int J Radiat Oncol Biol Phys. 2012 Jul;83(4):1101-6.

48 Kaufman EL, Jacobson JS, Hershman DL, Desai M, Neugut AI. Effect of breast cancer radiotherapy and cigarette smoking on risk of second primary lung cancer. J Clin Oncol. 2008 Jan;26(3):392-8

49 Ford MB, Sigurdson AJ, Petrulis ES, Ng CS, Kemp B, Cooksley C, et al. Effects of smoking and radiotherapy on lung carcinoma in breast carcinoma survivors. Cancer. 2003 Oct;98(7): 1457-64.

50 Johansen S, Cozzi L, Olsen DR. A planning comparison of dose patterns in organs at risk and predicted risk for radiation induced malignancy in the contralateral breast following radiation therapy of primary breast using conventional, IMRT and volumetric modulated arc treatment techniques. Acta Oncol. 2009;48(4):495-503.

51 Zurl B, Stranzl H, Winkler P, Kapp KS. Quantification of contralateral breast dose and risk estimate of radiation-induced contralateral breast cancer among young women using tangential fields and different modes of breathing. Int J Radiat Oncol Biol Phys. 2013 Feb;85(2):500-5.

52 Brenner AV, Preston DL, Sakata R, Sugiyama $\mathrm{H}$, de Gonzalez AB, French B, et al. Incidence of Breast Cancer in the Life Span Study of Atomic Bomb Survivors: 1958-2009. Radiat Res. 2018 Oct;190(4):433-44.

53 Kaiser JC, Jacob P, Meckbach R, Cullings HM. Breast cancer risk in atomic bomb survivors from multi-model inference with incidence data 1958-1998. Radiat Environ Biophys. 2012 Mar;51(1):1-14.

54 Marks LB, Zagar TM, Kaidar-Person O. Reassessing the time course for radiation-induced cardiac mortality in patients with breast cancer. Int J Radiat Oncol Biol Phys. 2017 Feb; 97(2):303-5.

55 Källman P, Agren A, Brahme A. Tumour and normal tissue responses to fractionated nonuniform dose delivery. Int J Radiat Biol. 1992 Aug;62(2):249-62.

56 Lee B, Lee S, Sung J, Yoon M. Radiotherapyinduced secondary cancer risk for breast cancer: 3D conformal therapy versus IMRT versus VMAT. J Radiol Prot. 2014 Jun;34(2):325-31.

57 van den Bogaard VA, Ta BD, van der Schaaf A, Bouma AB, Middag AM, Bantema-Joppe EJ, et al. Validation and Modification of a Prediction Model for Acute Cardiac Events in $\mathrm{Pa}$ tients With Breast Cancer Treated With Radiotherapy Based on Three-Dimensional Dose Distributions to Cardiac Substructures. J Clin Oncol. 2017 Apr;35(11):1171-8. 